\title{
Les Nouvelles sur la Russie dans les périodiques francophones et la propagande russe au début du règne de Pierre le Grand.
}

\author{
Nikolaï Kopanev \\ Bibliothèque nationale de Russie
}

\begin{abstract}
Резюме
Собранный автором материал позволяет оценить необходимость для России пропагандистских публикаций в западноевропейской прессе и понять основные направления приложения усилий литературных агентов, находившихся на службе России в царствование Петра I. Статья рассматривает становление русской пропаганды в первую половину петровской эпохи, включая и годы, предшествующие царствованию этого монарха. Особое внимание уделено двум основным аспектам этой пропаганды: публикации новостей о военных победах русской армии и о культурных преобразованиях в России, в особенности, публикации в России новых книг. Автор показывает также, кто был посредником между западноевропейской прессой и российскими властями. Отдельная часть статьи рассматривает историю публикации (а, точнее, задержки публикации) новостей о победе русской армии над шведами при Полтаве в официальном французском периодическом издании - “Gazette de France”.
\end{abstract}

Mots clé

presse francophone ; propagande ; Russie ; Hollande ; Pierre le Grand ; bataille de Poltava ; Gazette de France

Les premières victoires remportées par la Russie dans la guerre du Nord (prise de Schlusselbourg, de Nienschantz et de Narva) ont introduit le pays sur la " scène » de la grande politique européenne. La Russie eut aussi bien des alliés que des adversaires, qui utilisaient dans leur lutte les moyens les plus raffinés et les plus subtils de pression diplomatique. Dès cette époque, l'un des traits distinctifs de la diplomatie européenne était le désir de différents pays d'attirer l'opinion publique de leur côté et, dans la mesure du possible, de noircir leurs adversaires aux yeux des contemporains. Le meilleur moyen pour atteindre ce but était de publier des articles dans les journaux et dans les revues et aussi d'éditer des livres consacrés à tel ou tel événement politique. Il arrivait qu'une opération militaire ou la conclusion d'un traité fussent précédées de véritables "guerres de pamphlets » entraînant dans leur tourbillon une multitude de littérateurs à gages, des dizaines d'imprimeurs et les rédactions des périodiques les plus autorisés.

\section{Les premiers contacts avec la presse occidentale}

Les tsars russes commencèrent à s'intéresser à l'opinion publique européenne au milieu

\footnotetext{
${ }_{1}$ Ce texte a été préparé par Nikolaï Kopanev peu de temps avant sa mort. Son décès l'a empêché de terminer cet article. Nous avons néanmoins décidé de le publier en souvenir de l'auteur : même dans son état inachevé il témoigne de l'envergure de ses recherches et de sa manière propre d'explorer ses thèmes favoris tels que l'histoire du livre et de la presse, l'histoire intellectuelle et culturelle de la Russie du XVIII ${ }^{\mathrm{e}}$ siècle, notamment dans ses relations avec l'occident (n.d.r).
} 
du XVII ${ }^{\mathrm{e}}$ siècle, ou plus exactement, à partir de l'émergence du phénomène et de la notion d' " opinion publique européenne », étroitement liée au développement de l'édition et du journalisme. Il faut considérer l'affaire du «titre» du tsar Alexis Mikhailovitch, offense faite en 1666 par les "gazetiers d'Amsterdam et de Leyde », comme l'une des premières manifestations de l'attention accordée aux informations concernant la Russie et publiées dans les journaux et les revues d'Europe occidentale. A l'époque où les relations commerciales et politiques entre la Russie et les Pays-Bas étaient devenues très tendues, certains périodiques néerlandais commencèrent à appeler 2 le tsar russe simplement «Prince de Moscovie ». Le fait de diminuer l'importance du titre était loin d'être une erreur anodine des publicistes, car il réduisait d'emblée l'emprise territoriale de l'État russe et des possessions des Romanov, qui s'étendaient déjà des frontières de la Pologne jusqu'à celles de la Chine. L'intervention de l'ambassadeur russe et les protestations auprès de la partie hollandaise ne produisirent aucun effet jusqu'à ce que dix-huit marchands hollandais qui avaient vécu et fait du commerce à Moscou, ne soient intervenus en s'engageant officiellement par écrit à s'adresser au bourgmestre d'Amsterdam pour qu'il mît un terme à la manière trop libre dont les journalistes hollandais désignaient le tsar russe. Cet engagement, qui se conserva aux archives d'État, fut signé par un nombre important de marchands 3 .

Qui étaient-ils? Hendrich Thessing, par exemple, fut le représentant de la célèbre Maison des Thessing, qui entretenait à cette époque et au début du XVIII ${ }^{\mathrm{e}}$ siècle des relations commerciales très étroites avec la Russie. L'un des Tessing, Egberg, dirigeait une compagnie qui commerçait avec la Russie. Un autre Tessing, Friedrich, vivait habituellement à Vologda où il faisait le commerce du chanvre. Jan Tessing connut Pierre le Grand au cours de ses voyages à Moscou et à Arkhangelsk et, « lorsque Pierre était arrivé en Hollande, il avait été le premier à le reconnaître "4. Les intérêts des Tessing embrassaient des sphères lucratives dans le commerce franco-hollandais telles que le commerce du bois et d'autres marchandises, et les fournitures d'armement 5 . Jan Tessing intervenait également comme financier, «il prêtait de l'argent, se livrait à des opérations sur des lettres de change et sur des devises à la demande des grands seigneurs russes qui voyageaient avec Pierre $\mathrm{I}^{\mathrm{er}}$ et même acheminait le courrier diplomatique »6. Dès 16981699, pour plaire au tsar russe, Jan Tessing créa à Amsterdam une imprimerie et édita des œuvres en langue russe-slavonne commandées par Pierre le Grand. Au début de 1700, il se vit accorder une « charte » ou un «privilège » qui « ordonnait » au marchand hollandais

\footnotetext{
2 « Именовать » en langue russe.

3 Notamment par Werner Muller, Hendrick Boetenan, Hendrick Volckeringh, Jacob van der Heldt, Martin Bickling Marcuss, Daniel Hartmann, S. van den Tempel, Quikony de Wijse, Martin Ardinoys, Mateus Hopfler, Johannes de Remalmo, Hendr. Thessingh, Jan de Wilde, Abram Govars van Schyndel, Reynier Jansz Poll, Jacob van der Merxel et Dirk Double. Archives nationales des actes anciens de Russie (RGADA), fonds 5o, op. 1, (1666), d. 2, f ${ }^{\circ} 15^{-15}$.

4 П.П. Пекарский, Наука и литература в России при Петре Великом [P.P. Pékarski, Science et littérature en Russie sous Pierre le Grand, infra - Pékarski, 1862], Saint-Pétersbourg, 1862, t. I, p. 10.

${ }_{5}$ Par exemple, en 1698, ils demandèrent que l'on abaissât les taxes à l'exportation sur les bois de mâture en partance d'Arkhangelsk, en invoquant la forte concurrence de Narva et de Riga. En janvier 1698, Egberg Tessing finança à Lubeck l'achat de vingt-deux canons commandés pour la Russie et, en février de la même année, il rendit des services dans l'achat, pour l'armée russe, d'un grand lot d'armements dont le montant s'élevait à vingt mille roubles.

6 М.М. Богословский, Петр Великий. Материалы для биографии [M.M. Bogoslovski, Pierre le Grand. Documents pour sa biographie], Moscou, 1941, t. II, p. 226, 320, 359, 407.
} 
de «faire imprimer dans la ville d'Amsterdam » des cartes de Russie, dont les cartouches contenaient ordinairement le «Titre complet » des tsars de Russie et «toutes les sortes de feuilles imprimées et les personnages et ce qui avait trait aux hommes de guerre tant sur mer que sur terre », c'est-à-dire les portraits de Pierre le Grand et de ses proches. Le privilège portait aussi sur «des livres de mathématique, d'architecture et de ceux qui traitaient de la construction des villes et d'autres livres sur les arts [...] à l'exception des livres ecclésiastiques slaves de langue grecque »7. Ce privilège, qui donnait le monopole de l'édition des livres russes à Amsterdam à Jan Tessing, fut le premier document à définir les aspects fondamentaux des rapports entre les libraires-éditeurs de Russie et ceux d'Europe occidentale au XVIII ${ }^{\mathrm{e}}$ siècle. Il fut « somptueusement imprimé sur un parchemin dont les bords étaient parés d'or, d'argent et de vives couleurs ; son titre était orné des armoiries de la Russie et de celles des vingt-cinq autres régions qui dépendaient du tsar ; le sceau de l'État était suspendu à un cordon en or et toute la charte était recouverte d'étoffe de soie et de drap rouge $» 8$.

Les relations de la Russie avec les éditeurs et journalistes d'Europe occidentale furent entretenues au XVII ${ }^{\mathrm{e}}$ siècle notamment par l'intermédiaire du bourgmestre d'Amsterdam Nicolaas Witsen, qui était déjà venu en Russie sous le règne d'Alexis Mikhaïlovitch, et par celui d'André Vinius, maître des postes et chef du prikaz (ministère) de la Sibérie. En 1687, Witsen publia une carte géographique de l'Est et du Sud de l'Europe et de l'Asie qu'il dédia à Ioann et à Piotr Alexeïevitch, futur Pierre le Grand. Les jeunes tsars acceptèrent la dédicace avec bienveillance et envoyèrent à Witsen une lettre de remerciement sur laquelle était apposé le sceau de l'État. La même année, il fut ordonné à André Vinius de « faire imprimer outre-mer, en terre hollandaise », le portrait gravé de la Tsarevna Sophie, régente auprès de ses frères, les tsars Pierre et Ivan, en 1682-1689. Vinius fit expédier un modèle de gravure à Witsen et, peu après, il reçut d'Amsterdam une centaine d'effigies de la Tsarevna sur lesquelles était également indiqué son «titre complet et, en bas, on avait écrit en latin des vers à sa louange, où elle était appelée la Grande Souveraine [...] ces feuilles avaient été imprimées outre-mer afin qu'elle, la Grande Souveraine, en tirât gloire aussi bien outre-mer dans les autres états que dans l'état moscovite également »ı. Plus tard, à partir de 1695 , Vinius présentait presque chaque mois à Pierre $\mathrm{I}^{\mathrm{er}}$ des traductions et des extraits analytiques tirés de périodiques étrangersı.

\section{Pierre le Grand et la presse occidentale}

Le renforcement de la Russie suscita, au début du XVIII ${ }^{\mathrm{e}}$ siècle, le mécontentement dissimulé ou affiché de la majorité des pays d'Europe occidentale. Selon P.P. Pékarski, « les journalistes et publicistes européens parlaient de la Russie de l'époque de Pierre soit d'un ton persifleur, lorsqu'il était question de l'état intellectuel du pays, soit, lorsque l'on recevait une nouvelle des succès militaires du Tsar russe, avec des appréhensions semblables à la peur que suscitaient chez les Romains les bruits concernant les

\footnotetext{
${ }_{7}$ Полное собрание законов Российской империи [Collection complète des lois de l'empire de Russie], SaintPetersbourg, 1830, t. IV, $\mathrm{n}^{\circ}$ 1751, p. 6.

8 Pékarski, 1862, t. I, p. 11 ; Й. Дриссен, Царь Петр и его голландские друзья [J. Drissen, Le Tsar Pierre et ses amis hollandais], St.-Pétersbourg, 1996, p. 134. Ce parchemin s'est conservé jusqu'à nos jours et appartient aux descendants des Tessing. Il est aujourd'hui conservé au Gemeentearchief d'Amsterdam.

${ }_{9}$ Pékarski, 1862, t. I, p. 7.

1o Pékarski, 1862, t. I, p. 201-202.

п М.М. Богословский, Петр Великий, t. 1, р. 243, 247, 261, 289.
}

(cc) EY IRAARIFS 
barbares »12. Pierre $\mathrm{I}^{\mathrm{er}}$ lui-même et ses compagnons faisaient l'objet d'attaques dans les mémoires et dans la presse13.

Dès 1698, Pierre le Grand s'était réellement trouvé dans la nécessité de contrer les agents littéraires de Louis XIV. Dans le livre de l'agent du roi de France, Foy de la Neuville, Relation curieuse et nouvelle de Moscovie (Paris, 1698) le jeune monarque russe était présenté comme un despote oriental, un Néron du Nord, humiliant ses courtisans et admirant les incendies qui réduisaient régulièrement Moscou en cendre. François Lefort, illustre Genevois et compagnon d'armes du tsar russe, placé à la tête de sa Grande Ambassade dans les pays occidentaux, fit publier dans les journaux hollandais, allemands et suisses des échos bienveillants sur le voyage du tsar russe et s'impliqua directement dans la campagne contre le livre de La Neuville 14.

En 1702, Heinrich Freiherr von Hüyssen, homme d'Etat et de lettres et historien étroitement lié aux piétistes allemands, fut admis au service de la Russie. Selon son contrat, signé par Pierre $\mathrm{I}^{\mathrm{er}}$ en personne, il devait, en plus d'autres fonctions, traduire, faire imprimer et diffuser à l'étranger, les décrets du Tsar et persuader les savants de Hollande, d'Allemagne et d'autres pays, de consacrer leurs œuvres au Tsar, aux membres de sa famille, ou à des ministres du Tsar ${ }_{15}$. En 1705, Hüyssen fut envoyé en Allemagne où il s'efforça de faire publier un livre intitulé Relations sur la situation de l'Etat russe en 1703, mais aussi de convaincre Rabener, le rédacteur d'une revue européenne réputée, Europäische Fama, de publier des échos bienveillants sur la Russie. Et la même année, la revue publia des notices sur les progrès de l'instruction en Russie. On y développait en particulier une théorie progressiste sur le rapport et l'influence mutuels entre les cultures des peuples européens. En 1706, des articles semblables, écrits par Hüyssen, furent publiés dans la section « Nouvelles littéraires » d'un autre périodique réputé, les Mémoires pour l'histoire des sciences et des beaux-arts mieux connu sous le titre de Journal de Trévoux, qui paraissait en France.

Les Moscovites vont devenir sçavans, - lisait-on dans le numéro de la revue de juin 1706, - et les Muses semblent vouloir s'ouvrir une route vers la Tartarie. Vous sçavez qu'elles ont passé de l'Orient dans la Grèce, de la Grèce dans l'Italie, dans l'Espagne, dans la France, dans l'Allemagne, dans l'Angleterre et dans la Suède, où les sciences sont plus cultivées que jamais. Elles avancent vers le Nord, et le Czar Pierre Alexieuvits, c'est à dire fils d'Alexis, aujourd'hui regnant, a formé le dessein de chasser la barbarie de ses Etats. Après avoir parcouru l'Allemagne,_la Hollande et l'Angleterre et s'être instruit par son propre travail de l'Architecture Navale et Militaire, il a étendu ses soins jusqu'à l'instruction de ses sujetsı.

On parlait ensuite, dans cet article, de la création des écoles de jésuites allemands (des écoles allemandes) en Russie, «dans les faubourgs de Moscou », de l'école d'Ernst Glück, du Théâtre Anatomique de Bidloo, des écoles de navigation et de l'observatoire (« Tour

\footnotetext{
12 Pékarski, 1862, t. I, p. 90.

${ }_{13}$ Foy de La Neuville, Relation curieuse et nouvelle de Moscovie, Paris, 1698, 2 éd., La Haye, 1699. Un compte rendu en fut publié en 1699 dans le Journal des sçavans, t. XXVI, Amsterdam, 1699, juin, p. 416-421.

14 Н.А. Копанев, «Франц Лефорт, Вольтер, А.П. Веселовский », Швейцарцы в Петербурге [N.A. Kopanev, « François Le Fort, Voltaire, Abram Vesselovski », les Suisses à Saint-Pétersbourg], St.-Pétersbourg, 2002, p. 446-447.

${ }_{15}$ Pékarski, 1862, t. I, p.92.

${ }_{16}$ Mémoires pour l'histoire des sciences et des beaux-arts ou le Journal de Trévoux, Paris, 1706, juin, p. 10661068.
} 
Soukhariov»), de «l'admirable imprimerie de Moscou dont Fedor Polikarpov [était] devenu le directeur $\gg 17$. Il était aussi question des livres édités dans cette imprimerie18. Tel fut le début de la politique de publication de « bonnes nouvelles » sur la Russie.

L'influence militaire et diplomatique de la Russie rendait nécessaire l'établissement de relations entre les éditeurs-libraires d'Europe occidentale et la Russie. La consolidation de ces relations créait à son tour de nouvelles possibilités pour la diplomatie russe. C'est la raison pour laquelle, pendant le premier quart du XVIII ${ }^{\mathrm{e}}$ siècle, les liens de la Russie avec les éditeurs se développèrent non seulement grâce aux efforts d'agents littéraires comme Hüyssen, engagés spécialement à cette fin, mais aussi grâce à ceux des membres officiels des missions russes à l'étranger : ambassadeurs, secrétaires d'ambassades, consuls et agents commerciaux qui, pour remplir leurs fonctions, devaient recourir à des éditeurs et imprimeurs prêts à publier immédiatement, dans n'importe quel pays européen, des brochures ou notices expliquant la politique de la Russie.

Très significative à cet égard fut l'action de l'un des diplomates russes les plus en vue à l'époque de Pierre, le premier ambassadeur extraordinaire et plénipotentiaire de Russie en Hollande, Andreï Artamonovitch Matveev. L'histoire de la diplomatie a retenu que, dès 1700, lorsque la nouvelle de la défaite de Narva était arrivée à La Haye, il avait rédigé et remis au gouvernement hollandais un mémoire qui «devait dissiper la mauvaise impression produite par cette nouvelle »; de plus, l'ambassadeur de Suède avait dû donner un démenti publicı. Des préoccupations incomparablement plus agréables échurent au même Matveev neuf ans plus tard, lorsqu'il eut à informer l'Europe Occidentale de la victoire des armées russes près de Poltava. On le sait, aussitôt après la débâcle de l'armée de Charles XII, plusieurs relations furent écrites sur cette victoire, qui furent expédiées en Hollande et remises par le ministre plénipotentiaire russe au président des États-Généraux. L'une de ces relations, rédigée par Pierre $\mathrm{I}^{\mathrm{er}}$ en personne, fut immédiatement traduite en français et éditée en Hollande sous forme de brochure. Bientôt, un plan de la bataille de Poltava parut à son tour. Matveev prit soin que ces détails fussent reproduits sur le cuivre par un graveur hollandaiszo. Le plan fut imprimé à La Haye, par l'éditeur Pierre Husson, en grand format et avec des explications en

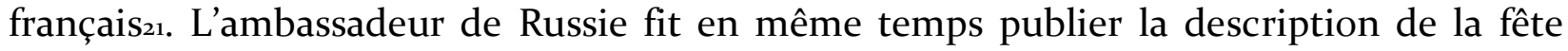
grandiose et du feu d'artifice qu'il avait organisés à La Haye en l'honneur de la victoire de

${ }_{17}$ Ibid.

18 On y parlait surtout du livre de Stefan Iavorski: Стефан Яворский, Знамения пришествия Антихристова и кончина века [Stefan Iavorski, Les signes de la venue de l'Antéchrist et de la fin des temps], Moscou, 1704. Ce travail réfutait un manuscrit de Grigori Talitski, diffusé en copies manuscrites, dans lequel on tentait de démontrer que la fin du monde était arrivée et que Pierre I ${ }^{\mathrm{er}}$ était l'Antéchrist. Dans le même numéro de la revue, on annonçait la parution du Dictionnaire slavo-gréco-latin de Fedor Polikarpov (Федор Поликарпов, Славяно-греко-латинский словарь, Moscou, 1704), ainsi que d'un Nouveau manuel d'arithmétique (Новый спосо6 арифметики, Moscou, 1705, premier livre russe imprimé où le nom de Copernic fut mentionné).

${ }_{19}$ История дипломатии [Histoire de la diplomatie], sous la direction de V.P. Potiomkine, Moscou, 1941, t. I, p. 275.

zо А. Круазе-ван-дер-Коп, «Как отнеслась Голландия к победе русских под Полтавой », ЖМНП [Anna Croiset van der Kop, "Comment la Hollande réagit à la victoire des Russes près de Poltava ", Revue du ministère de l'Education nationale], 1910, partie 27, $\mathrm{n}^{\circ}$ 5, p. 125-126.

${ }_{21}$ Plan de la fameuse bataille, donnée aux environs de Poltava en Ucraine, entre l'armée de Sa Majesté Cézarienne Pierre I Empereur de la Grande Russie et celle de S. M. le Roi de Suède Charles XII, le 27/8 juillet 1709, La Haye, Pierre Husson. 
Poltava et sur l'ordre de Pierre $\mathrm{I}^{\mathrm{er}}{ }_{22}$. Matveev avait en Hollande, dès cette époque, un réseau bien au point d'intermédiaires dans l'édition.

C'est précisément à cette époque que l'on se mit à publier des articles bienveillants sur la Russie. Puis, dans les années 1710 , les orientations fondamentales de la collaboration entre la Russie et les journalistes d'Europe occidentale trouvèrent de nouveaux développements. Dans les meilleures revues, on publia des articles sur la culture de la Russie, sur les réformes de Pierre $\mathrm{I}^{\mathrm{er}}$, sur les progrès de l'édition russe 23 . En août 1709 , un mois après Poltava, on pouvait lire dans la réédition que l'on faisait à Amsterdam de la revue française le Journal des savants : «Malgré le tumulte que la guerre cause en ce Royaume [en Russie - N.K.], le Czar a fait faire en Hollande de nouveaux caractères moscovites et de nouvelles Presses d'imprimerie qu'il a fait apporter à Moscou au commencement de l'année 1708. Ces nouveaux caractères approchent fort des caractères gothiques ; et depuis que ces presses roulent, on a déjà publié les livres suivans en langue moscovite ». Venait ensuite l'énumération des neuf premiers livres imprimés à Moscou en nouveaux caractères civils 24 . En effet, en 1707 , sur l'ordre de Pierre $\mathrm{I}^{\mathrm{er}}$, on fabriqua à Amsterdam des «caractères russes nouvellement inventés » (les caractères civils) qui furent bientôt apportés en Russie avec deux presses en état de marche et tout le nécessaire pour les faire fonctionner et avec d'autres matériels d'imprimerie. La même année, on recruta en Hollande plusieurs maîtres imprimeurs qui, pour une rémunération assez élevée, avaient accepté de partir en Russie. L'année suivante, en 1708, parut à Moscou le premier livre russe imprimé en nouveaux caractères civils, Geometriâ slavenski zemlemerie [Géométrie, en slave: zemlemerié, Moscou, 1708). «Un grand nombre de bons livres composez ou traduits depuis peu en langue Esclavonne qui est la langue naturelle du pays, prouvent que les Moscovites ne sont pas loin d'acquérir par les Sciences autant de gloire que par les Armes », - lisons-nous dans le numéro de février 1710 du Journal des savants. L'auteur de la notice ne manquait pas de rappeler sur le champ que «tous ces livres [avaient] été imprimés avec des caractères que le Prince [avait] fait venir de Hollande »25. Or, tout porte à croire que cet auteur était Ilia Fedorovitch Kopiévitch, un compagnon de Tessing, engagé en 1698 par Pierre le Grand pour rédiger et éditer à Amsterdam plusieurs livres sur des sujets laïques. Dès décembre 1699, ce littérateur avait une vingtaine d'œuvres prêtes à être mises sous presse à l’imprimerie de Tessing 26 . Après le décès de

${ }_{22}$ Relation des festins que S. E. André de Matveof ... a célébré par ordre de son Maître à la Haye en réjouissance de la fameuse bataille de Poltawa, S. 1., 1709.

${ }_{23}$ C'est ainsi qu'entre 1710 et 1714 , le Journal de Trévoux publia par trois fois dans ses pages des listes de livres édités dans les imprimeries de Moscou et de Pétersbourg. Н.A. Копанев, « Петр I - переводчик », XVIII век, vol. 16. Итоги и проблемы изучения русской литературы XVIII века [N.A. Kopanev, «Pierre I ${ }^{\mathrm{er}}$ traducteur ", XVIII siècle, vol. 16, Bilan et questions de l'étude de la littérature russe du XVIII siècle], Leningrad, 1989, p. 180-183.

${ }_{24}$ Journal des sçavans, 1709, t. 45, p. 428-431.

${ }_{25}$ Mémoires pour l'histoire des sciences et des beaux-arts... Paris, 1710, févr., t. XXXVII, p. 352, 353. Dans cette notice et dans les suivantes, on découvre qui furent les auteurs de certaines œuvres publiées alors anonymement en Russie et, en particulier, il s'avère que Правило о пяти чинех архитектурфы Бороццо да Виньолы [La règle des cinq ordres de l'architecture de Borozzo da Vinioli, Moscou, 1709], le premier livre en langue russe sur l'architecture, fut traduit par Pierre I ${ }^{\text {er }}$ lui-même. Ces données sont aussi confirmées par une autre notice parue en 1711 dans le Journal de Trévoux : « il a fait traduire et fait imprimer plusieurs livres écrits avec beaucoup de discernement, et lui même n'a pas dédaigné d'en traduire quelques-uns » (Mémoires pour l'histoire des sciences et des beaux-arts..., 1711, septembre, t. 43, p. 1657).

${ }_{26}$ Pékarski, 1862, t. I, p. 14-15. 
Tessing en 1701, Kopiévitch seul continua l'édition des livres russes en Europe. En 1703, il fut nommé traducteur de l'ambassade russe à Copenhague et, en 1704, il proposa à August Hermann Francke à continuer dans sa typographie à Halle la série des publications ordonnées par Pierre le Grand, qui pouvait servir à l'éducation de la jeunesse russe 27 . Plus tard, en 1710, Kopiévitch fut nommé au Collège des Affaires étrangères russes28. Dans les « Nouvelles littéraires de Moscovie » publiées dans le Journal de Trévoux en 1711, nous trouvons une « liste des livres publiés par Monsieur Kopiévitch en langue slavonne »29 et encore une notice sur la Moscovie dans le numéro de décembre $17143^{\circ}$.

Une information aussi abondante sur les éditions russes dans les périodiques francophones faisait connaître les réformes fondamentales de Pierre $\mathrm{I}^{\mathrm{er}}$ dans le domaine de la culture et soutenait le prestige du titre du tzar de Russie, en le présentant comme un monarque éclairé.

\section{La victoire de Poltava rapportée par la Gazette de France}

Doit-on considérer ces efforts de propagande du côté russe comme disproportionnés ou abusifs ? Essayons de répondre à cette question en explorant la couverture donnée au principal événement de la première période de la guerre du Nord, la bataille de Poltava, dans la Gazette de France, le périodique français alors le plus connu, qui se trouvait sous la protection du roi. Commençons par le numéro du 3 août 1709 dans lequel fut imprimée une lettre de Hambourg datée du 19 juillet 1709, qui ne disait pas un seul mot de la victoire de l'armée russe, dix jours avant, mais dans laquelle on pouvait lire :

De Hambourg, le 19 Juillet 1709

Les nouvelles des armées du Roy de Suéde et du Czar, ainsi que celles de Pologne, continuënt d'estre non seulement fort incertaines, mais mesme souvent elles se contredisent. On assuroit que le Roy de Suéde estoit du costé de Pereaslaw prés du Borysthene ; et les derniers avis portent qu'il continuoit le siege de Pultawa, à quinze lieuës de cette riviere, et à prés de quarante de Pereaslaw : que les assiegez commençoient à manquer de poudre, et que le Czar qu'on disoit estre revenu à son armée, avoit ordonné à ses Generaux de tout hazarder pour y en faire entrer : ce qui ne s'accorde pas avec le mauvais estat auquel on publioit que l'armée suédoise estoit reduite, puisque celle du Czar, qu'on assuroit estre beaucoup superieure, et qu'elle tenoit les suédois comme assiegez, n'entreprenoit pas de leur donner bataille, quoyqu'elle eut construit plusieurs ponts sur le Worskla qui passe à Pultawa ${ }_{31}$.

Deux semaines plus tard, le 17 août, la Gazette de France publiait une autre lettre de Hambourg, du 2 août 1709 :

27 Eduard Winter, Halle als Ausgangspunkt der deutschen Rußlandkunde im 18. Jahrhundert, Berlin, 1953, S. 396-397.

28 Н.А. Копанев, « Петр I - переводчик », art. cit., p. 180-183.

${ }_{29}$ Mémoires pour l'histoire des sciences et des beaux-arts..., 1711, septembre, t. 43, p. 1657-166o.

$3_{0}$ Dans cet article, après les discours habituels où il était dit que le Tsar « avait fait fleurir les lettres dans un païs sterile jusqu'ici pour elles» et que, «sur cette voie, même les alarmes de la guerre» ne l'avaient pas entravé, on racontait les succès de l'imprimerie de Moscou « qui [produisait] tous les ans de bons livres » et l'on donnait la liste de huit nouveaux livres imprimés en Russie. Les titres de ces livres étaient écrits en translittération latine et traduits en français. Mémoires pour l'histoire des sciences et des beaux-arts..., 1714, décembre, t. 56, p. 2171.

${ }_{31}$ Gazette de France, 3 août 1709, n 31, p. 361-362. 
[...] Le 28 du passé juillet, un courrier de ce Prince du Roy Auguste passa en cette ville, allant à Coppenhague [sic!], porter, suivant le bruit commun, la nouvelle qu'il avoit receuë de Cracovie, de la défaite entiere de l'armée Suédoise par les Moscovites, envoyée par le General Goltz, avec la lettre que le Czar luy avoit écrite du 27 juin, auquel jour la bataille s'estoit donnée : et que la mesme nouvelle avoit este confirmée par des lettres du 22 juillet, d'Olmutz en Moravie et de Breslau en Silesie. Ce grand évenement trouve d'autant moins de creance, que les diverses copies qui ont esté répanduës de cette lettre du Czar au General Goltz, sont differentes pour le stile et pour les circonstances : que la confirmation et les particularitez que le Czar, suivant cette lettre, promettoit d'envoyer incessamment, ne sont point arrivées, quoyque les courriers de ce Prince ayent la liberté des passage : que le Baron de Stralenheim qui est à Breslau, n'en a eu aucun avis : que des lettres posterieures de Cracovie n'en font aucune mention ; et enfin, que plusieurs autres nouvelles publiées cidevant, des avantages considerables remportez par les Moscovites sur les Suédois, se sont trouvées fausses, ce qui est cause que cette lettre du Czar n'a pas esté ici renduë publique. D’ailleurs, les lettres de Dantzig du 27 juillet, ou l'on auroit du plustost en estre informé, n'en parlent en aucune maniere ; et elles portent au contraire, que le Roy de Suéde avoit pris Pultowa. Les lettres de Varsovie, de Cracovie et de Caminietz du 20 et du 21 juillet, gardent pareillement le silence sur ce sujet... Il y a aussi des lettres de Reval et de Riga en Livonie, du 22 jullet, qui portent que le Roy de Suéde avoit pris Pultowa d'assaut, et battu les Moscovites à Voronitz. Quoyqu'il en soit, il est peu vraysemblable que depuis le 27 juin, il n'en fust pas venu quelque confirmation, si la nouvelle dela defaite entiere de l'armee suedoise par les Moscovites estoit veritable z2. $^{2}$

On voit que presque un mois après la bataille de Poltava, la publication de la relation officielle russe sur cette victoire était restée interdite à Hambourg. En même temps, on accordait toute l'attention aux fausses nouvelles sur la prise de Poltava par Charles XII. Le numéro suivant de la Gazette publiait un fragment de la relation officielle russe sur la victoire de Poltava :

Il paroist ici et en plusieurs autres villes d'Allemagne, une copie entiere, imprimée à Dresde, de la lettre ecrite par ordre du Czar au General Goltz, touchant la bataille gagnée par les Moscovites contre les Suédois, dont il a esté déja parlé. On en attend encore la confirmation parce que la lettre est datée du lendemain de la bataille, et que par consequent c'est la mesme qu'on avoit veuë ci-devant, si ce n'est qu'elle est plus ample et contient plus de circonstances. Voici en substance ce qu'elle porte, en attendant qu'on puisse en estre mieux ëclairci par des lettres plus seures et plus recente.

Elle marque d'abord, que la bataille s'estoit donné le 8 juillet, c'est à dire le 27 juin, selon le vieux stile que suivent les Moscovites. L'armée du Czar traversa la Worskla le 4 juillet, et elle s'avança à un quart de lieuë des Suédois, où elle se retrancha, ayant plusieurs défilez devant elle. Le 8. à la pointe du jour, la cavalerie suédoise, suivie de l'infanterie, passa les défilez et attaqua la cavalerie moscovite, qui fut poussée jusqu'aux retranchemens, d'où elle revint à la charge et rompit l'â̂le droite des suédois. En mesme temps le Czar envoya le Prince Menzikow avec un corps considerable, pour attaquer le corps de reserve des suédois, composé de trois mille hommes postez prés d'un bois, pour couvrir leur â̂le droite. Il les defit, et revint ensuite a l'armée, aprés avoir détaché le general Rentzel pour attaquer le general Rosen, qui estoit resté devant Pultowa, et qui fut aussi défait. Cependant le Czar fit mettre son infanterie en bataille sur deux lignes, en laissant une troisiéme pour la garde des retranchements. Sur les neuf heures, les suédois s'avancerent et

${ }_{32}$ Gazette de France, 17 août 1709, n 33 , p. 385-386. 
recommencerent le combat, dans lequel leur infanterie fut enfoncée et obligée à se retirer en desordre, poursuivie par les moscovites jusqu'à la nuit. Le Czar détacha le Prince Galiczen, le general Baur avec de nombreuses troupes de cavalerie et de dragon, suivis par le Prince Menzikow, pour tacher d'attendre les suédois. On ajoute qu'il n'y avoit aucune nouvelle du Roy de Suede, et qu'on croyoit qu'il s'etoit retiré avec six mille chevaux et ce qui s'estoit pû sauver de son infanterie : que le nombre des suédois tuez en cette bataille estoit de huit ou dix mille hommes, et celui des prisoniers de prés de trois mille, parmi lesquels estoient le Comte Piper Secretaire d'Etat, le General Renschild, les Generaux Majors Slippenbach, Stakelberg, Rosen et Hamilton, et un grand nombre d'autres Officiers : enfin que les Moscovites avoient pris cent trente sept drapeaux ou estendars, les chariots, les bagages et quatre pieces de canon. Voila de quelle maniere le succes de ce combat est rapporté dans cette lettre, qui ne fait aucune mention de la perte des moscovites, dont elle promettoit de marquer le détail au bas de la relation 33 .

Peu de temps après, le journaliste français eut de nouveau des doutes sur la victoire des armées russes. Le 31 août 1709, plus d'un mois après la bataille de Poltava, quand des cérémonies officielles célébrant cet événement ont déjà eu lieu à Moscou et à La Haye, la Gazette écrivait à ses lecteurs :

[...] Plusieurs Senateurs estoient partis de Cracovi pour venir trouver ce Prince [Le Roy de Pologne], sur la nouvelle qui s'estoit repanduë que l'armée suédoise avoit esté défaite par les Moscovites. Ce bruit continuë encore ; mais quoyqu'on en ait publié à Dresde une relation fort circonstanciée, l'Electeur de Brandenbourg n'a pas voulu permettre qu'on l'imprimast à Berlin : ce qui donnoit un nouveau sujet d'en douter. Il paroist depuis peu une seconde relation du 11 juillet, de la défaite entiere du reste de l'armée suédoise eschapé de la bataille, au nombre d'environ seize mille hommes. Elle porte que plusieurs détachements des troupes du Czar commandez par le Prince Menzikow, avoient atteint les Suédois à Perewolozna sur les bords du Borysthene: que n'ayant point de pont pour traverser cette riviere, et manquant de vivres et de Munitions, ils avoient esté contraints le 11 juillet, de se rendre par une capitulation comprise en sept articles [...] qu'ensuite ils s'etoient rendus au nombre de seize mille deux cent quatre-vingt sept, sans y comprendre plusieurs domestiques de la Maison du Roy de Suéde, avec cent quarante deux drapeaux ou estendarts, vingt-un petits canons de bronze, deux de fer, et huit mortier aussi fort petits. On ajoute que le Roy de Suede avoit passé le Borysthene avec trois cent chevaux choisis [...] Tant de particuliaritez feroient donner une entiere creance à ces nouvelles, quoyqu'elles ayent toutes esté publiées par le General Goltz, si elles n'estoient contredites par des lettres venuës par diverses voyes, qui donnent beaucoup de sujet d'en douter. Le Maitre de la Poste de Stetin a receu une lettre du Sieur Biber Secretaire du Cabinet du Roy de Pologne, écrite de Boleslaw dans le Palatinat de Sandomir, du 25 juillet, par laquelle il lui mande qu'on avoit esté extremement touché de la nouvelle repanduë par les ennemis, de la defaite entiere et mesme de la mort du Roy de Suéde : mais que ce jour-la 25 juillet, le Roy son Maitre venoit d'en recevoir une toute contraire, qui estoit que les Moscovites avoient perdu trente mille hommes en voulant tenter le secours de Pulowa, et que la place avoit esté prise avec sept mille hommes qui en composoient la garnison [...] Une autre lettre de Konigsberg du 26 juillet, porte ce qui suit. Nous avons receu ce matin de meilleurs nouvelles du Roy de Suéde, dont les troupes aprés avoir esté repoussées quatre fois, devant Pultowa, l'avoient emporté au cinquieme assaut et passé au fil de l'espée sept mille Moscovites qui estoient dedans. Le Czar a tenté de les secourir, mais il a esté receu de maniere que presque toute son infanterie a esté ruinée, et il ne s'est sauvé que la moindre partie de sa cavalerie. Enfin, le Baron de Stralenheim écrit de Breslau en Silesie du 8 aoust, au Ministre Suédois qui est à

${ }_{33}$ Gazette de France, 24 août 1709, n 34, p. 398-399. 
Francfort, qu'il venoit de recevoir un courrier du Roy de Suéde, qui lui mandoit que les Moscovites avoient esté entierement défaits : qu'ils avoient perdu en plusieurs combats durant quatre jours, plus de soixante mille hommes, avec les bagages et l'artillerie : que dix mile Suedois avoient esté tuez : que le Roy de Suéde se porte bien, et qu'il avoit six mille Moscovites prisonniers. Ces nouvelles sont si differentes les unes des autres, qu'on ne peut y ajoûter foy, jusqu'à ce qu'on en ait eu la confirmation et de plus grands éclaircissements, qu'on ne peut pas tarder à recevoir, soit par le retour du Roy de Suéde, ou par de nouveaux courriers $_{34}$.

Le 7 septembre 1709, soit deux mois après la bataille de Poltava, la Gazette de France dut reconnaître la défaite des Suédois. Voici l'extrait de cette publication datée du 23 août 1709, de Hambourg :

Le 19 août, Le Sieur Westofski Secretaire de la Chancellerie du Czar, arriva en cette ville Hambourg, allant à Copenhague, porter au Roy de Danemark la confirmation de la nouvelle qu'on avoit ci-devant receuë, de la bataille gagnée prés de Pultowa le 8 juillet, par les Moscovites, dans laquelle les Suédois avoient eu huit mille hommes tuez, et les troupes du Czar seulement huit cent huit hommes morts, et trois mille deux cent quatre-vingt-trois blessez. Il rapporte aussi que le 11 juillet, le General Lewenhaupt avoit esté obligé de se rendre avec le reste de l'armee Suédoise, au Prince Menzikow : que le mesme jour, le Roy de Suede avoit passé le Borysthene, avec six à sept cent cavaliers choisis ; et que se voyant poursuivi par un détachement de Moscovites, il s'estoit retiré à Oczakow à l'embouchure de la mesme riviere, ou il avoit esté tres-bien receu par le Bacha qui y commande. Ce courrier ajoûte, qu'il avoit demeuré cinq semaines en chemin, ayant passé par la Transylvanie, la Hongrie et la Silesie, pour éviter les lieux infectez de la contagion, et éviter d'estre arresté par les troupes de Roy de Pologne. Ces nouvelles ont esté confirmées par des lettres du 17 juillet de Moscou, où il en a esté fait de grandes rejouïssances, et de divers endroits de Pologne : de maniere que peu de gens doutent de cet évenement, exepté de quelques circonstances 35 .

C'est un cas très curieux qui montre à quel point les fausses nouvelles de la défaite de l'armée russe à Poltava, soutenues par certains périodiques français, pouvaient être persistantes bien que les Russes eussent connu à Poltava une victoire complète sur l'armée de Suède.

Ces exemples montrent que les efforts de propagande de Pierre le Grand et de ses collaborateurs, qui voulaient faire connaître au public occidental les succès des armes et des lettres russes, n'étaient pas inutiles. Les relations des périodiques francophones avec les agents littéraires de Pierre le Grand passaient à cette époque souvent par l'intermédiaire des piétistes allemands et par les chefs de compagnies commerçantes de Hollande, qui furent les premiers à soutenir les réformes du tsar russe au début de son règne 36.

\footnotetext{
${ }_{34}$ Gazette de France, 31 août 1709, n 35, p. 410-412.

${ }_{35}$ Gazette de France, 7 septembre $1709, \mathrm{n}^{\circ}$ 36, p. 421-422.

${ }_{3}$ Amsterdam, Pierre Humbert, 1720-1740. Voir Н.A. Копанев, « Реклама русских изданий за границей в первой половине XVIII в. ", Книга в Pоссии XVI-XIX в. [N.A. Kopanev, «L'annonce à l'étranger de publications russes dans la première moitié du XVIII ${ }^{\mathrm{e}}$ siècle ", Le Livre en Russie au XVI ${ }^{e}-X I X^{e}$ siècles],
} 
Après le décès d'Ilia Kopievitch (septembre 1714), la tradition de la publication des "Nouvelles littéraires» de Moscou et de Saint-Pétersbourg fut continuée par l'intermédiaire de la Gazette de France qui fit écho aux différents aspects du voyage de Pierre le Grand en France 37 , mais aussi par les rédacteurs de la Bibliothèque germanique, ou histoire littéraire de l'Allemagne, de la Suisse et des Pays du Nord éditée par Jacques Lenfant, Louis de Beausobre, Paul-Emile de Mauclerc et J.-H.-S. Formey. 